\section{(6) OPEN ACCESS}

\title{
Impact of early disease factors on metabolic syndrome in systemic lupus erythematosus: data from an international inception cohort
}

\author{
Ben Parker, ${ }^{1,2}$ Murray B Urowitz, ${ }^{3}$ Dafna D Gladman, ${ }^{3}$ Mark Lunt, ${ }^{1,2}$ Rachelle Donn, \\ Sang-Cheol Bae, ${ }^{4}$ Jorge Sanchez-Guerrero, ${ }^{5}$ Juanita Romero-Diaz, ${ }^{5}$ Caroline Gordon, \\ Daniel J Wallace, ${ }^{7}$ Ann E Clarke, ${ }^{8}$ Sasha Bernatsky, ${ }^{8}$ Ellen M Ginzler, ${ }^{9}$ \\ David A Isenberg, ${ }^{10}$ Anisur Rahman, ${ }^{10}$ Joan T Merrill, ${ }^{11}$ Graciela S Alarcón, ${ }^{12}$ \\ Barri J Fessler, ${ }^{12}$ Paul R Fortin, ${ }^{13}$ John G Hanly, ${ }_{1}^{14}$ Michelle Petri, ${ }^{15}$ \\ Kristjan Steinsson, ${ }^{16}$ Mary Anne Dooley, ${ }^{17}$ Susan Manzi, ${ }^{18}$ Munther A Khamashta, ${ }_{1}{ }^{19}$ \\ Rosalind Ramsey-Goldman, ${ }^{20}$ Asad A Zoma, ${ }^{21}$ Gunnar K Sturfelt, ${ }^{22}$ Ola Nived, ${ }^{22}$ \\ Cynthia Aranow, ${ }^{23}$ Meggan Mackay, ${ }^{23}$ Manuel Ramos-Casals, ${ }_{1}{ }^{24}$ Ronald F van \\ Vollenhoven, ${ }^{25}$ Kenneth C Kalunian, ${ }^{26}$ Guillermo Ruiz-Irastorza, ${ }^{27}$ S Sam Lim, ${ }^{28}$ \\ Diane L Kamen, ${ }^{29}$ Christine A Peschken, $^{30}$ Murat Inanc, ${ }^{31}$ Ian N Bruce ${ }^{1,2}$
}

\begin{abstract}
Handling editor Tore K Kvien
- Additional material is published online only. To view please visit the journal online (http://dx.doi.org/10.1136/ annrheumdis-2013-203933).

For numbered affiliations see end of article.
\end{abstract}

\section{Correspondence to} Professor lan N Bruce, Arthritis Research UK Centre for Epidemiology, Centre for Musculoskeletal Research, Institute of Inflammation and Repair, Manchester Academic Health Science Centre, The University of Manchester, Oxford Road, Manchester M13 9PT, UK:

ian.bruce@manchester.ac.uk

Received 12 May 2013 Revised 10 March 2014 Accepted 16 March 2014 Published Online First 1 April 2014

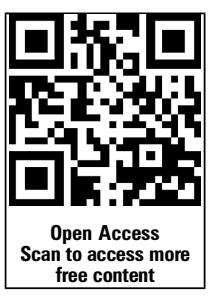

CrossMark

To cite: Parker $B$, Urowitz MB, Gladman DD, et al. Ann Rheum Dis 2015;74:1530-1536.

\section{ABSTRACT}

Background The metabolic syndrome (MetS) may contribute to the increased cardiovascular risk in systemic lupus erythematosus (SLE). We examined the association between MetS and disease activity, disease phenotype and corticosteroid exposure over time in patients with SLE.

Methods Recently diagnosed ( $<15$ months) patients with SLE from 30 centres across 11 countries were enrolled into the Systemic Lupus International Collaborating Clinics (SLICC) Inception Cohort from 2000 onwards. Baseline and annual assessments recorded clinical, laboratory and therapeutic data. A longitudinal analysis of factors associated with MetS in the first 2 years of follow-up was performed using random effects logistic regression.

Results We studied 1150 patients with a mean (SD) age of 34.9 (13.6) years and disease duration at enrolment of 24.2 (18.0) weeks. In those with complete data, MetS prevalence was $38.2 \%$ at enrolment, 34.8\% at year 1 and $35.4 \%$ at year 2 . In a multivariable random effects model that included data from all visits, prior MetS status, baseline renal disease, SLICC Damage Index $>1$, higher disease activity, increasing age and Hispanic or Black African race/ethnicity were independently associated with MetS over the first 2 years of follow-up in the cohort.

Conclusions MetS is a persistent phenotype in a significant proportion of patients with SLE. Renal lupus, active inflammatory disease and damage are SLE-related factors that drive MetS development while antimalarial agents appear to be protective from early in the disease course.

\section{INTRODUCTION}

Women with systemic lupus erythematosus (SLE) have a greater than fivefold increased risk of clinical coronary heart disease (CHD) events ${ }^{1}$ and an increased burden of subclinical atherosclerosis, as measured by coronary calcium, carotid plaque, arterial stiffness and endothelial dysfunction. ${ }^{2-5}$ Although classic Framingham risk factors are more prevalent in SLE, ${ }^{6}$ they do not fully explain this excess CHD risk.

The metabolic syndrome (MetS) is a clustering of related CHD risk factors associated with an increased cardiovascular risk in the general population, ${ }^{8} 9$ and is a useful clinical tool to identify patients who may warrant more focused CHD risk assessment. ${ }^{10}$ MetS is also more prevalent in SLE than in matched control populations, ${ }^{11}$ and may therefore contribute to the pro-atherogenic environment in SLE. We have previously shown in a cross-sectional analysis of patients with recently diagnosed SLE that MetS occurred in 36.4\% of patients. In this study, MetS was associated with Black African, Korean and Hispanic race/ethnicity, a more severe lupus phenotype and exposure to high-dose corticosteroids. ${ }^{12}$ The impact of disease-related factors on MetS development over time is not yet understood and may differ over the course of the disease. Understanding the interplay between disease activity, therapeutic exposure and MetS in SLE would better inform CHD risk stratification and help guide treatment regimens in higher risk patients.

The Systemic Lupus International Collaborating Clinics (SLICC) group has developed an inception cohort to facilitate prospective longitudinal studies of risk factors for the development of atherosclerosis in SLE. Using this inception cohort, we aimed to investigate the factors associated with MetS in patients with SLE over the first 2 years of follow-up in the SLICC Inception Cohort.

\section{METHODS}

SLICC Inception Cohort and definition of MetS

The methodology used in this study has been described previously. $^{12}$ In brief, the SLICC 
Inception Cohort was established between 2000 and 2009 from 30 centres in 11 countries in North America, Europe and Asia. Patients were enrolled when $\geq 4$ American College of Rheumatology (ACR) classification criteria for SLE $^{13}$ were recognised, and all patients were enrolled within 15 months of the date of their diagnosis. There were no other specific exclusion criteria. MetS was defined according to the 2009 definition described in the Joint Interim Statement from the International Diabetes Federation Task Force on Epidemiology and Prevention and interested partners. ${ }^{14}$

SLE disease activity and damage were assessed using the Systemic Lupus Erythematosus Disease Activity Index $\left(\right.$ SLEDAI-2K) ${ }^{15}$ and the SLICC/ACR Damage Index (SDI), ${ }^{16}$ respectively. Clinical features and locally performed laboratory tests (fasting or non-fasting) to assess disease activity, evaluate CHD risk factors and define MetS were submitted annually to the coordinating centre at the University of Toronto. Disease phenotype data (anti-dsDNA antibody, complement and thrombocytopenia data) were also extracted from the SLEDAI-2K.

\section{Statistical analysis}

Potential SLE factors implicated in MetS development were defined a priori. These represented inflammatory disease activity (SLEDAI-2K), disease phenotype (such as active renal disease, thrombocytopenia, high anti-dsDNA antibodies) and therapeutic exposures including several measures of corticosteroid exposure (including current dose, past doses and length of course, intravenous use/dose, peak dose received between visits and total cumulative dose). Comparison of continuous data was performed using Wilcoxon's rank sum test or paired t test, and of categorical data using $\chi^{2}$ test. Variation of MetS over time was also assessed in 399 patients with complete 2-year data. In our longitudinal analysis we included all cases in whom a MetS status could be determined at any time point, using random effects logistic regression to account for repeated measures within individuals. Multiple regression analyses, adjusted for age, gender and race/ethnicity and the varying prevalence of MetS over time, were performed initially. Variables were generated to examine the differential effect of recorded exposures over time (enrolment vs follow-up). Interactions between predictor variables and between predictor variables and follow-up were tested for and included in the multivariable model where relevant. Factors that were significant on initial adjusted analyses $(\mathrm{p}<0.2)$ were subsequently entered into a multivariable random effects model. The results presented are adjusted OR and 95\% CIs. All statistical analyses were performed using STATA V.10.0.

\section{RESULTS}

\section{Patients}

Patient characteristics are summarised in table 1. At enrolment, the mean (SD) disease duration was 24.2 (18.0) weeks, 1036/ 1150 (90.1\%) patients were women and there was a wide racial/ ethnic variation, reflecting the geographical distribution of participating centres: 516/1148 (45.0\%) were Caucasian, 182/1148 (15.9\%) were Hispanic, 154/1148 (13.4\%) were black African or Afro-Caribbean and 151/1148 (13.2\%) were Korean. All patients satisfied the 1997 modified ACR criteria for SLE ${ }^{13}$ and $95.5 \%$ of the cohort had a positive ANA. Sufficient data were available to define the presence or absence of MetS in 1150/ 1686 patients $(68.2 \%)$ at enrolment, $823 / 1211$ patients $(68.0 \%)$ at year 1 and 686/1021 patients (67.2\%) at year 2. Fasting lipid data were the most frequent missing item. No significant differences in age, gender, damage/activity indices, renal disease or medication use at any time point were noted in patients with missing MetS status. However, significant differences were noted in race/ethnicity and country of origin in patients with missing MetS status. For example, at baseline 79/158 (50\%) patients of African ancestry had missing MetS status compared with 20/171 (11.7\%) Korean subjects, 75/257 (29.2\%) Hispanics and 244/516 (37.8\%) Caucasian subjects. These differences remained stable over follow-up.

\section{Prevalence of MetS over time}

Overall, MetS was present in 439/1150 (38.2\%) at enrolment, $286 / 823(34.8 \%)$ at year 1 and 243/686 (35.4\%) at year 2 . The prevalence of MetS varied significantly over time according to race/ethnicity (figure 1). For example, in Caucasians it occurred in $35.5 \%, 32.6 \%$ and $31.4 \%$ at baseline, year 1 and year 2 , respectively, while in patients of African ancestry the prevalence of MetS prevalence at each visit was 57\%, 38.8\% and $62 \%$ and in Koreans it was $41.7 \% 29.2 \%$ and $32.8 \%$, respectively. In a complete case analysis of the 399 patients with a documented MetS status at all three visits, $186 / 399(46.6 \%)$ of patients had MetS on at least one occasion, 88 (22.1\%) had MetS at every visit, $62(15.6 \%)$ developed incident MetS during follow-up and 213 (53.4\%) never developed MetS (figure 2).

\section{Factors associated with MetS over time}

Using random effects logistic regression, we tested the strength of the association between individual predefined disease-related variables and MetS over the first 2 years of follow-up in the whole cohort $(n=1150$ at enrolment). These analyses were adjusted for age, race/ethnicity, gender and the varying prevalence of MetS over time. As can be seen in table 2, higher disease activity and SDI scores, renal lupus and higher oral doses of corticosteroids were all associated with MetS. Although numbers were small, intravenous corticosteroid use (eg, at enrolment 52 patients treated with intravenous corticosteroids, 28 of whom received $\geq 3$ 'pulses') also showed a trend towards being associated with MetS (OR 1.60, 95\% CI 0.87 to 2.97). Current antimalarial (AM) use was associated with a reduced prevalence of MetS over the first 2 years of follow-up (OR 0.21, 95\% CI 0.14 to 0.34 ). Having MetS at the previous visit was most strongly associated with prevalent MetS (OR 18.6, 95\% CI 13.7 to 25.4).

The strength of association between several exposures and $\mathrm{MetS}$ varied according to timing of exposure. For example, the association between immunosuppressant exposure and MetS was very strong at baseline but less so over the subsequent 2 years (OR 8.04, 95\% CI 4.53 to 14.3 vs OR 2.66, 95\% CI 1.47 to 4.76 ). A similar pattern was seen with peak oral corticosteroid dose (per mg) (OR 1.07, 95\% CI 1.05 to 1.09 vs OR $1.03,95 \%$ CI 1.01 to 1.04 ). All univariate results are available in online supplementary tables S1-S3.

In a final multivariable random effects model we found that prior MetS status, baseline renal disease, any damage (SDI $>1$ ), higher disease activity, increasing age and Hispanic or African ancestry race/ethnicity remained independently associated with MetS over the first 2 years of follow-up. AM use was protective against MetS development (table 3). When we excluded preceding MetS status from the model, baseline immunosuppressant use (OR 2.10, 95\% CI 1.09 to 4.06), higher peak corticosteroid dose at baseline (OR 1.04, 95\% CI 1.03 to 1.06), AM use over follow-up (OR $0.27,95 \%$ CI 0.14 to 0.54 ), SDI $>1$ over follow-up (OR 5.24, 95\% CI 2.53 to 10.9) and active renal disease over follow-up (OR 3.62, 95\% CI 1.45 to 9.03) were associated with MetS, in addition to increasing age and African ancestry and Korean race/ethnicity. Hispanic race/ethnicity did not remain in this exploratory model. 
Table 1 Characteristics of patients in the SLICC Inception Cohort over first 2 years*

\begin{tabular}{|c|c|c|c|}
\hline & Enrolment & Year 1 & Year 2 \\
\hline No. of patients & 1150 & 823 & 686 \\
\hline Age (years) (mean (SD)) & $34.9(13.6)$ & $36.2(13.7)$ & $37.2(13.9)$ \\
\hline \multicolumn{4}{|l|}{ Gender (\%) } \\
\hline Women & $1036(90.1)$ & $729(88.6)$ & $608(88.6)$ \\
\hline \multicolumn{4}{|l|}{ Race/ethnicity (\%) } \\
\hline Caucasian & $516(44.9 \%)$ & $399(48.5)$ & $347(50.6 \%)$ \\
\hline Indian subcontinent & $37(3.2)$ & $39(4.7)$ & $21(3.1)$ \\
\hline Black African & $79(6.9)$ & $49(6.0)$ & $50(7.3)$ \\
\hline Afro-Caribbean & $75(6.5)$ & $61(7.4)$ & $41(6.0)$ \\
\hline Korean & 151 (13.2) & $106(12.9)$ & $67(9.8)$ \\
\hline Hispanic & $182(15.9)$ & $89(10.8)$ & $83(12.1)$ \\
\hline Other & $108(9.4)$ & $80(9.7)$ & $77(11.2)$ \\
\hline \multicolumn{4}{|l|}{ CHD risk factors (mean (SD)) } \\
\hline BP systolic (mm Hg) & $118.5(16.4)$ & $117.5(16.8)$ & $117.8(16.3)$ \\
\hline BP diastolic (mm Hg) & 74.7 (10.7) & $73.9(10.5)$ & 73.5 (10.6) \\
\hline On AHT medication (\%) & $328(28.5)$ & $259(31.5)$ & $246(35.9)$ \\
\hline Total cholesterol $(\mathrm{mmol} / \mathrm{L})$ & $4.89(1.50)$ & $4.59(1.11)$ & $4.57(1.12)$ \\
\hline Triglyceride (mmol/L) & $1.78(1.21)$ & $1.45(1.1)$ & $1.39(0.95)$ \\
\hline HDL-cholesterol (mmol/L) & $1.39(0.61)$ & $1.44(0.49)$ & $1.43(0.47)$ \\
\hline Lipid-lowering medication (\%) & $168(14.6)$ & $138(16.8)$ & $124(18.1)$ \\
\hline Glucose $(\mathrm{mmol} / \mathrm{L})$ & $5.02(1.71)$ & $4.78(1.00)$ & $4.74(1.05)$ \\
\hline Smoker current (\%) & $169(14.7)$ & $113(13.8)$ & $97(14.2)$ \\
\hline Premenopausal (\%) & $813(70.6)$ & $558(67.8)$ & $464(67.6)$ \\
\hline BMI & $24.8(5.9)$ & $25.4(5.9)$ & $25.2(6.1)$ \\
\hline WC $(\mathrm{cm})$ & $82.0(14.0)$ & $83.3(14.9)$ & $82.3(14.5)$ \\
\hline SLEDAI (mean (SD)) & $5.4(5.2)$ & $3.7(41)$ & $3.7(4.2)$ \\
\hline SLICCIACR-DI $\geq 1$ (\%) & $97 / 504(19.3)$ & $215 / 815(26.4)$ & 208/679 (30.6) \\
\hline \multicolumn{4}{|l|}{ Disease phenotype (\%) } \\
\hline Active renal disease & $261(22.8)$ & $132(16.2)$ & 93 (13.6) \\
\hline Anti-dsDNA positive & 427/1034 (41.3) & $262 / 766(34.2)$ & $227 / 672(33.8)$ \\
\hline Low complement & 419/1038 (40.4) & 273/766 (35.6) & 229/672 (34.1) \\
\hline Oral CS use (\%) & 796 (69.2) & 581 (70.6) & $401(58.5)$ \\
\hline \multicolumn{4}{|l|}{ Oral CS dose (median (IQR)) } \\
\hline Average CS dose(mg) & $20(10,34)$ & $10(7,15)$ & $8.0(5,12.5)$ \\
\hline Highest CS dose(mg) & $40(20,60)$ & $20(10,40)$ & $10(5,20)$ \\
\hline Cumulative CS dose (g) & $2.6(1.0,5.0)$ & $3.8(2.5,6.1)$ & $5.6(3.7,8.9)$ \\
\hline Pulse IV CS (\%) & $52 / 1095(4.9)$ & $57 / 819(7.0)$ & $24 / 683(3.5)$ \\
\hline Immunosuppressant use (\%) & $464(40.4)$ & $337(41.0)$ & $299(43.6)$ \\
\hline Azathioprine & $196(43.7)$ & $141(42.0)$ & $126(42.3)$ \\
\hline Methotrexate & $104(17.4)$ & 62 (18.6) & $63(21.1)$ \\
\hline Mycophenolate mofetil & 98 (16.4) & 65 (19.5) & 59 (19.6) \\
\hline IV cyclophosphamide & $95(15.9)$ & $35(10.4)$ & $29(9.9)$ \\
\hline Cyclosporin & $21(3.5)$ & $16(4.7)$ & $14(4.8)$ \\
\hline Other & $19(3.2)$ & $17(4.1)$ & $8(2.6)$ \\
\hline Antimalarial use (\%) & $759(66.0)$ & $555(67.4)$ & $483(70.4)$ \\
\hline
\end{tabular}

Regarding race/ethnicity, almost all patients of Korean race/ ethnicity resided in South Korea $(165 / 169 ; 97.6 \%)$, the majority of Hispanic patients resided in Mexico (192/240; 80\%) or the USA $(39 / 240 ; 16.3 \%)$ and most patients of African ancestry resided in either the USA $(69.2 \%)$ or Europe (20.5\%). As shown in figure 1, the overall prevalence of MetS varied over time within each racial/ethnic group, but was substantially lower over the follow-up period in Koreans compared with baseline. Figure 3 describes the significant variation in MetS phenotype over time in those of African ancestry, Korean and Hispanic race/ethnicity compared with the whole cohort. Full characteristics by race/ethnicity are available in online supplementary table S4.

\section{DISCUSSION}

MetS was common in the SLICC Inception Cohort over the first 2 years after enrolment, ranging between $34.8 \%$ and $38.2 \%$ overall. Despite a high proportion of patients having persistent MetS at each visit, variation remained in the MetS status of many individuals over time. For example, over the 2-year 


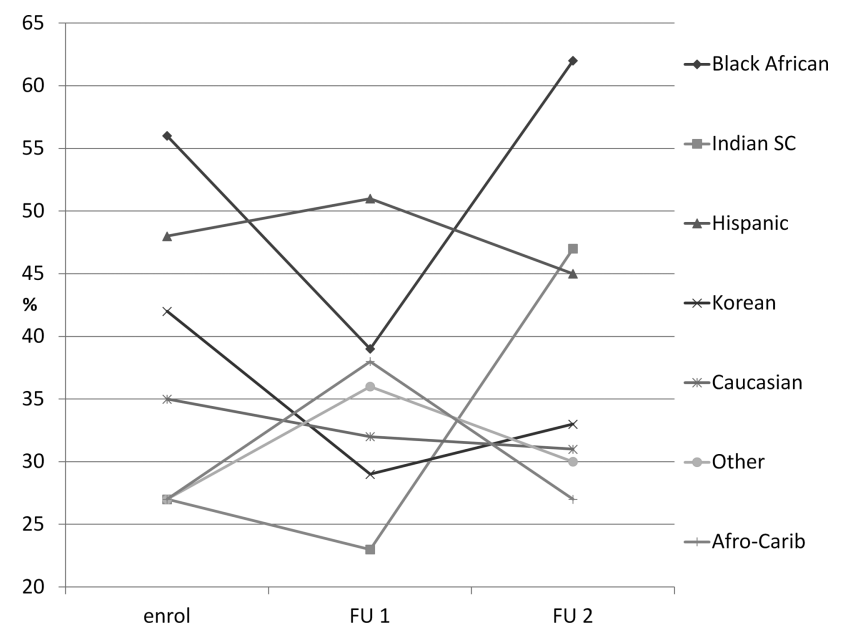

Figure 1 Prevalence of metabolic syndrome over time by race/ ethnicity. FU, follow-up.

follow-up period, $15.6 \%$ developed incident MetS and overall $46.6 \%$ of patients had MetS on at least one occasion. Whether persistent or transitory MetS in a young patient with SLE is associated with future cardiovascular events is a key question currently under investigation within this cohort, and will help to further validate the use of MetS as a cardiovascular risk prediction tool in SLE.

As in our baseline analysis, ${ }^{12}$ we also found significant racial/ ethnic variation in MetS prevalence over time. The highest prevalence was noted in Hispanic patients and those of African ancestry at each visit. Korean patients had a high baseline prevalence $(41.7 \%)$ which reduced over time $(29.2 \%$ at year 1 and $32.8 \%$ at year 2), while Caucasian patients had a relatively stable prevalence at each visit. To some degree, this racial/ethnic variation in MetS prevalence reflects the background prevalence of MetS in different populations ${ }^{9} 1718$ and the reported higher rates of MetS in geographically diverse SLE cohorts compared with their local matched controls. ${ }^{19-23}$ However, the contrasting MetS and SLE phenotype observed in these racial/ethnic SLE populations suggests that central obesity may not be the key driving factor in all patients in all races/ethnicities. For example, Hispanic patients and those of African ancestry had persistently
Table 2 Significant factors associated with MetS over time in SLICC Inception Cohort in age, race/ethnicity, gender and time adjusted univariate analyses*

\begin{tabular}{lcc}
\hline Variable & Adjusted OR & $95 \%$ Cl \\
\hline Age (years) & 1.08 & 1.06 to 1.11 \\
Previous MetS status & 18.6 & 13.7 to 25.4 \\
African ancestry race/ethnicity & 8.11 & 2.69 to 24.4 \\
Hispanic race/ethnicity & 5.17 & 2.28 to 11.7 \\
SLEDAI-2K>10 & 2.26 & 1.54 to 3.32 \\
SLEDAI-2K (per unit) & 1.11 & 1.07 to 1.16 \\
SLICCIACR-DI>1 & 7.84 & 4.32 to 14.2 \\
Active renal diseaset & 7.31 & 4.47 to 11.9 \\
Current oral CS & 3.94 & 2.38 to 6.55 \\
Average oral CS dose $(\mathrm{mg}) \ddagger$ & 1.06 & 1.05 to 1.08 \\
Highest oral CS dose $(\mathrm{mg}) \ddagger$ & 1.04 & 1.03 to 1.05 \\
Cumulative oral CS dose $(\mathrm{g})$ & 1.11 & 1.07 to 1.16 \\
Current immunosuppressant & 2.06 & 1.42 to 3.00 \\
Current antimalarial & 0.21 & 0.14 to 0.34
\end{tabular}

*All variables are assessed as present or absent unless otherwise stated. tDefined as haematuria $>5$ red blood cells/high power field; pyuria $>5$ white blood cells/high power field; new or recent increase of $>500 \mathrm{mg} 24 \mathrm{~h}$ protein; casts including granular or red blood cells; or consistent renal biopsy; nephrotic syndrome (proteinuria $>3 \mathrm{~g} / 24 \mathrm{~h}$, oedema and increased BP). Other causes excluded. ¥Within preceding 12 months.

CS, corticosteroid; MetS, metabolic syndrome; SLEDAI, Systemic Lupus Erythematosus Disease Activity Index; SLICCIACR-DI, Systemic Lupus Erythematosus International Collaborating Clinics/American College of Rheumatology Damage Index.

high rates of central obesity but Koreans had a high baseline prevalence of MetS (which declined over time) despite the lowest prevalence of central obesity. Corticosteroid use was almost universal in the Korean population, with frequent early use of intravenous methylprednisolone, but this group had comparable oral corticosteroid doses to the rest of the cohort. An important question prompted by this observation is whether the high baseline MetS prevalence in the Korean population in part reflects a more inflammatory MetS phenotype, ${ }^{19}$ and therefore high intravenous/oral corticosteroid use gave rapid control of active disease and improved inflammation-related metabolic derangements (and hence reduced MetS). In contrast, long-term steroid use in Hispanics and those of African ancestry, with a
Figure 2 Persistence and variability of metabolic syndrome (MetS) over time in a complete case analysis $(n=399)$. FU, follow-up
MetS Persistence ( $n=399)$

$\underline{\text { FU } 1}$

FU 2

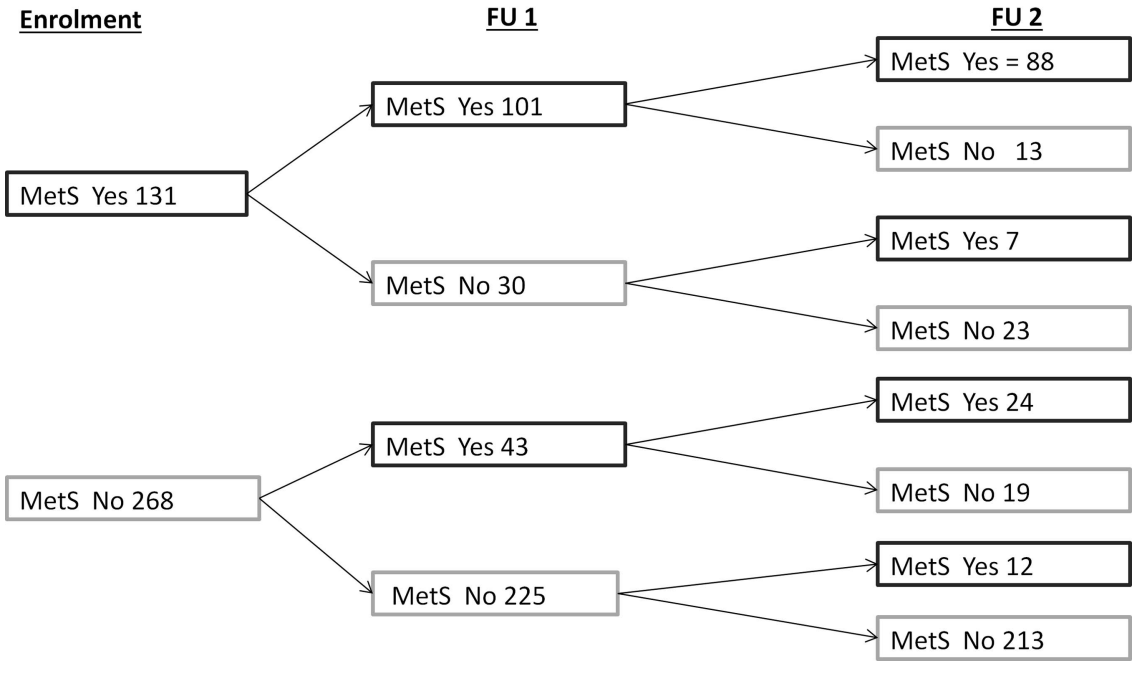


Table 3 Final multivariable random effects model of clinical associations of MetS over time

\begin{tabular}{lcc}
\hline Variable & Adjusted OR & $95 \% \mathrm{Cl}$ \\
\hline MetS at previous visit $(y / n)$ & 14.9 & 10.7 to 20.8 \\
Renal disease at baseline $(y / n)$ & 1.53 & 1.01 to 2.3 \\
Antimalarial over time $(y / n)$ & 0.67 & 0.47 to 0.95 \\
SLICC/ACR-DI>1 over time $(y / n)$ & 2.37 & 1.64 to 3.42 \\
SLEDAI over time (per unit increase) & 1.07 & 1.02 to 1.13 \\
Age (years) & 1.04 & 1.03 to 1.05 \\
Hispanic & 2.25 & 1.28 to 3.96 \\
African ancestry & 3.35 & 1.59 to 7.01
\end{tabular}

MetS, metabolic syndrome; SLEDAI, Systemic Lupus Erythematosus Disease Activity Index; SLICCIACR-DI, Systemic Lupus Erythematosus International Collaborating

Clinics/American College of Rheumatology Damage Index.

propensity to central obesity, may have a detrimental effect on the evolution and persistence of MetS. It is likely that genetic factors may underlie some of these differences by influencing the SLE phenotype and/or by affecting an individual's sensitivity to the effects of corticosteroids.

Our longitudinal study found that factors independently associated with MetS over the first 2 years of follow-up in patients with early SLE include 'fixed' factors such as African ancestry and Hispanic race/ethnicity and age, as well as potentially modifiable factors such as renal disease at enrolment, higher SLEDAI-2K and the presence of damage on the SDI. Preceding MetS was most strongly associated with prevalent MetS over the first 2 years of follow-up, and our complete case analysis confirmed that a substantial proportion of patients with MetS at baseline had persistent MetS at each subsequent visit. Data to assess whether MetS predates the diagnosis of SLE in these patients were not collected within the SLICC Inception Cohort.

The association with active lupus, lupus nephritis and damage suggests that the SLE inflammatory process may contribute to MetS over time in this population. A more 'inflammatory' MetS phenotype not chiefly driven by central obesity was suggested by previous studies. ${ }^{11} 19$ Parker et al noted that low C3 complement was associated with MetS, and other cross-sectional clinical studies have also noted associations between aspects of the inflammatory phenotype and MetS in SLE cohorts, such as previous nephritis and higher SLEDAI scores ${ }^{24}$ as well as raised C-reactive protein. ${ }^{25}$ In addition, data from lupus-prone mouse models suggest that MetS and insulin resistance may develop prior to the actual onset of clinically overt disease. ${ }^{26}$ Inflammation and progression of SLE may therefore have a key role in driving MetS over time. The lack of association with steroid use over time in our final model is of interest. We cannot exclude an important contribution of steroids to MetS in SLE and, indeed, in our univariate analysis as well as our exploratory analysis where we excluded MetS status from the model, both suggest an important contribution of steroids to this phenotype. Overall, from a therapeutic point of view, our data suggest that prompt and focused suppression of disease activity, particularly nephritis, is likely to have a significant effect on future development of MetS in patients with SLE, particularly if this can be achieved with minimal chronic steroid exposure. Early measures to control and modulate MetS and cardiovascular risk should therefore be an additional therapeutic goal in SLE, and recent experience in renal transplantation has demonstrated that 'steroid-free' regimes can be developed in situations where potent and reliable immunosuppression is clearly required. This approach has also been proposed in lupus nephritis, ${ }^{27}$ and our data suggest that such an approach may have the added benefit of modifying the long-term risk of cardiovascular disease in SLE.

The independent 'protective' effect of AMs on MetS development over time observed in this study may reflect the numerous atheroprotective effects of AMs observed in other studies (such as on lipid profile and insulin resistance ${ }^{28}{ }^{29}$ ) and positive effects on disease stability and steroid regimes. There still remains the possibility that their apparent protective effect on MetS reflects persistent confounding, such as selection bias, although this is minimised through the prospective study design and longer follow-up in our cohort.

This is the largest study to date examining MetS in SLE and has many advantages over previous studies. First, it is the only
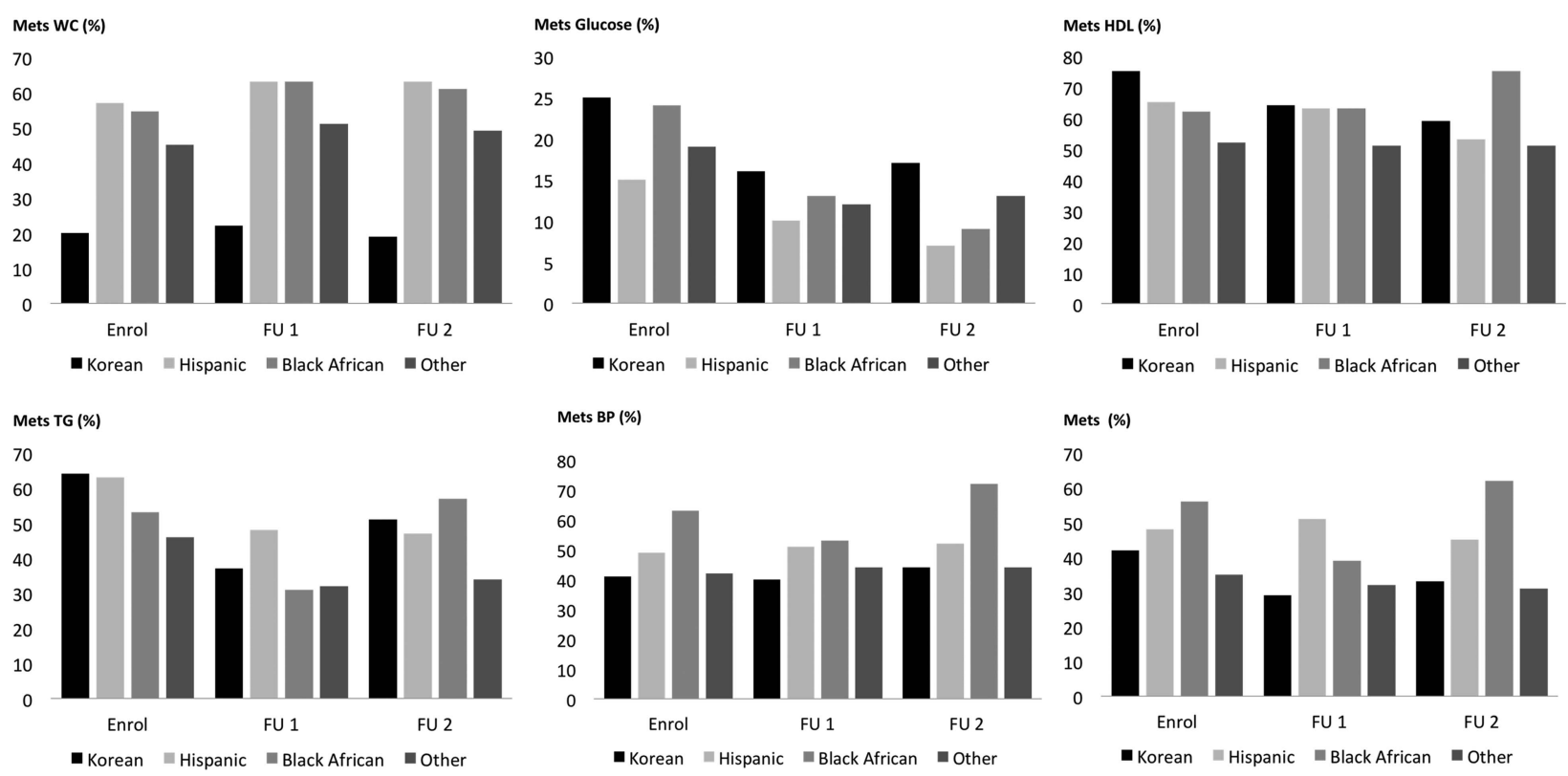

Figure 3 Metabolic syndrome (MetS) characteristics by race/ethnicity. BP, blood pressure; FU, follow-up, HDL, high-density lipoprotein; TG, triglyceride; WC, waist circumference. 
study that has examined the determinants of MetS over time and the prospective nature of the cohort limits many potential sources of bias associated with retrospective studies. Second, the cohort is young and has a range of disease activity that allows detailed exploration of the impact of inflammation on MetS development. Also, the most recent definition of MetS has been used, which recognises that patients without central obesity can meet the definition. The effects of non-obesity-related factors on MetS development could therefore be explored, unlike studies that use definitions of MetS 'anchored' by central obesity. The SLICC cohort is international and recruited from centres in 11 countries, with a range of racial/ethnic groups and socioeconomic backgrounds, and therefore the results can be generalised to a wide range of SLE populations. Finally, and perhaps uniquely, a broad range of detailed data on corticosteroid dosing were captured which permitted detailed analyses of the effect of corticosteroids on MetS, a weakness of existing studies.

The analysis does, however, have several limitations. First, there are missing MetS data in many patients and particularly those in the highest risk race/ethnicity group (African ancestry), a potential source of bias. Second, the use of MetS as a CHD risk prediction tool has yet to be validated in SLE and is the focus of ongoing work. Finally, there is no control population against which to compare the prevalence of MetS, which hinders the interpretation of the results. While population level data are available for most participating countries, population cohorts are generally older with a higher proportion of men than the SLICC cohort, so direct comparisons cannot be made. However, all controlled studies to date have found that MetS is more common in SLE than in age-matched controls. ${ }^{11}$

Our study found that the risk of developing MetS could be determined early in the SLE disease course. This clustering of CHD risk factors and the observed racial/ethnic variation in MetS susceptibility should help inform risk stratification in individual patients and improve the personalised management of early disease. Lupus nephritis in very early disease, persistent disease activity and the evolution of damage over time all significantly influence the development of MetS, which is a persistent phenotype in a substantial number of patients. From disease onset, therapeutic regimes should aim to rapidly control active disease and should include AMs. Corticosteroid doses should be individually tailored in order to minimise longer term cardiovascular risk, especially in high-risk populations.

\footnotetext{
Author affiliations

${ }^{1}$ Arthritis Research UK Centre for Epidemiology, Centre for Musculoskeletal Research, Institute of Inflammation and Repair, Manchester Academic Health Sciences Centre, The University of Manchester, Manchester, UK

${ }^{2}$ NIHR Manchester Musculoskeletal Biomedical Research Unit, Central Manchester University Hospitals NHS Foundation Trust, Manchester Academic Health Science Centre, Toronto, Ontario, Canada

${ }^{3}$ Centre for Prognosis Studies in the Rheumatic Diseases, Toronto Western Hospital and University of Toronto, Toronto, Ontario, Canada

${ }^{4}$ Department of Rheumatology, Hanyang University Hospital for Rheumatic Diseases, Seoul, Korea

${ }^{5}$ Instituto Nacional de Ciencias Medicas y Nutrición, Mexico City, Mexico

${ }^{6}$ Rheumatology Research Group, School of Immunity and Infection, College of Medical and Dental Sciences, University of Birmingham, Birmingham, UK ${ }^{7}$ Cedars-Sinai/David Geffen School of Medicine at UCLA, Los Angeles, California, USA

${ }^{8}$ Divisions of Clinical Immunology/Allergy and Clinical Epidemiology, Montreal General Hospital, McGill University Health Centre, Montreal, Quebec, Canada ${ }^{9}$ Department of Medicine, SUNY Downstate Medical Center, Brooklyn, New York, USA

${ }^{10}$ Centre for Rheumatology Research, University College, London, UK

${ }^{11}$ Department of Clinical Pharmacology, Oklahoma Medical Research Foundation, Oklahoma City, Oklahoma, USA

${ }^{12}$ Division of Clinical Immunology and Rheumatology, Department of Medicine, University of Alabama at Birmingham, Birmingham, Alabama, USA
}

${ }^{13}$ Division of Rheumatology, Centre Hospitalier Universitaire de Québec et Université Laval, Quebec City, Quebec, Canada

${ }^{14}$ Division of Rheumatology, Department of Medicine and Department of Pathology, Queen Elizabeth II Health Sciences Centre and Dalhousie University, Halifax, Nova

Scotia, Canada

${ }^{15}$ Department of Rheumatology, Johns Hopkins University School of Medicine, Baltimore, Maryland, USA

${ }^{16}$ Center for Rheumatology Research, Landspitali University hospital, Reykjavik, Iceland

${ }^{17}$ Division of Rheumatology and Immunology, Department of Medicine, University of North Carolina, Chapel Hill, North Carolina, USA

${ }^{18}$ Lupus Center of Excellence, Allegheny Health Network, Pittsburgh, Pennsylvania, USA

${ }^{19}$ Lupus Research Unit, The Rayne Institute, St Thomas' Hospital, King's College London School of Medicine, London, UK

${ }^{20}$ Northwestern University and Feinberg School of Medicine, Chicago, Illinois, USA

${ }^{21}$ Lanarkshire Centre for Rheumatology, Hairmyres Hospital, East Kilbride, UK

${ }^{22}$ Department of Rheumatology, University Hospital Lund, Lund, Sweden

${ }^{23}$ Feinstein Institute for Medical Research, Manhasset, New York, USA

${ }^{24}$ Department of Autoimmune Diseases, Josep Font Autoimmune Diseases

Laboratory, IDIBAPS, Hospital Clínic, Barcelona, Spain

${ }^{25}$ Unit for Clinical Therapy Research (ClinTRID), The Karolinska Institute, Stockholm, Sweden

${ }^{26}$ UCSD School of Medicine, La Jolla, California, USA

${ }^{27}$ Autoimmune Disease Unit, Department of Internal Medicine, Hospital Universitario Cruces, University of the Basque Country, Barakaldo, Spain

${ }^{28}$ Emory University, Atlanta, Georgia, USA

${ }^{29}$ Medical University of South Carolina, Charleston, South Carolina, USA

${ }^{30}$ University of Manitoba, Winnipeg, Manitoba, Canada

${ }^{31}$ Division of Rheumatology, Department of Internal Medicine, Istanbul Medical

Faculty, Istanbul University, Istanbul, Turkey

Contributors The study was conceived by INB, ML, MBU and DDG. Data analysis and interpretation and manuscript preparation was performed by BP, MBU, DDG, $\mathrm{ML}$ and INB. All authors contributed to data collection, critically reviewed and edited the manuscript and approved the final version.

Funding This study was funded by the Canadian Institutes of Health Research (grant number 93695), Arthritis Research UK (Arthritis Research UK Epidemiology Unit Core Support Programme Grant) and independent research supported by the National Institute for Health Research (NIHR) Biomedical Research Unit Funding Scheme and the NIHR Manchester Biomedical Research Centre (BP, ML, INB, RD). INB is a NIHR Senior Investigator and is supported by Arthritis Research UK, the Manchester Academic Health Science Centre, the NIHR Biomedical Research Unit Funding Scheme, the NIHR Manchester Wellcome Trust Clinical Research Facility and the NIHR Manchester Biomedical Research Centre. Additional author support: BP (Arthritis Research Clinical Research Fellowship 18845); S-CB (Korea Healthcare Technology R\&D project (A120404), Ministry for Health and Welfare, Republic of Korea); CG (Lupus UK, NIHR/Wellcome Trust Clinical Research Facility at University Hospital Birmingham NHS Foundation Trust and City Hospital, Sandwell and West Birmingham Hospitals NHS Trust, UK); RR-G (NIH grants UL1 RR025741, P60AR 30692, K24 AR 002138); MP (Hopkins Lupus Cohort NIH grant RD-1 43727); GR (Department of Education, Universities and Research, Basque Government); SB (Singer Family Fund for Lupus Research); MAD (NIH grant RR00046); PRF (tier 1 Canada Research Chair on Systemic Autoimmune Rheumatic Diseases, Université Laval); JGH (Canadian Institutes of Health Research, operating grant number 86526). The views expressed in this publication are those of the authors and not necessarily those of the NHS, the NIHR or the Department of Health.

\section{Competing interests None.}

Ethics approval The study was approved by the University Health Network Research Institute research ethics committee, Toronto, Canada and by the Institutional Research Ethics Boards of all participating centres in accordance with the Declaration of Helsinki's guidelines for research in humans.

Provenance and peer review Not commissioned; externally peer reviewed.

Open Access This is an Open Access article distributed in accordance with the Creative Commons Attribution Non Commercial (CC BY-NC 3.0) license, which permits others to distribute, remix, adapt, build upon this work non-commercially, and license their derivative works on different terms, provided the original work is properly cited and the use is non-commercial. See: http://creativecommons.org/ licenses/by-nc/3.0/

\section{REFERENCES}

1 Manzi S, Meilahn EN, Rairie JE, et al. Age-specific incidence rates of myocardial infarction and angina in women with systemic lupus erythematosus: comparison with the Framingham Study. Am J Epidemiol 1997;145:408-15. 
2 Petri MA, Kiani AN, Post W, et al. Lupus Atherosclerosis Prevention Study (LAPS). Ann Rheum Dis 2011;70:760-5.

3 Ahmad $Y$, Shelmerdine J, Bodill $H$, et al. Subclinical atherosclerosis in systemic lupus erythematosus (SLE): the relative contribution of classic risk factors and the lupus phenotype. Rheumatology 2007;46:983-8.

4 Roman MJ, Shanker BA, Davis A, et al. Prevalence and correlates of accelerated atherosclerosis in systemic lupus erythematosus. N Engl J Med 2003:349:2399-406.

5 El-Magadmi M, Bodill H, Ahmad Y, et al. Systemic lupus erythematosus: an independent risk factor for endothelial dysfunction in women. Circulation 2004; 110:399-404

6 Bruce IN, Urowitz MB, Gladman DD, et al. Risk factors for coronary heart disease in women with systemic lupus erythematosus: the Toronto Risk Factor Study. Arthritis Rheum 2003;48:3159-67.

7 Esdaile JM, Abrahamowicz M, Grodzicky T, et al. Traditional Framingham risk factors fail to fully account for accelerated atherosclerosis in systemic lupus erythematosus. Arthritis Rheum 2001:44:2331-7.

8 Alberti KG, Zimmet P, Shaw J. Metabolic syndrome - a new world-wide definition. A Consensus Statement from the International Diabetes Federation. Diabet Med 2006;23:469-80.

9 Cornier MA, Dabelea D, Hernandez TL, et al. The metabolic syndrome. Endocr Rev 2008:29:777-822.

10 Expert Panel on Detection, Evaluation, and Treatment of High Blood Cholesterol in Adults. Executive Summary of the Third Report of The National Cholesterol Education Program (NCEP) Expert Panel on Detection, Evaluation, and Treatment of High Blood Cholesterol in Adults (Adult Treatment Panel III). JAMA 2001;285:2486-97.

11 Parker $B$, Bruce IN. The metabolic syndrome in systemic lupus erythematosus. Rheum Dis Clin North Am 2010:36:81-97.

12 Parker B, Urowitz MB, Gladman DD, et al. Clinical associations of the metabolic syndrome in systemic lupus erythematosus: data from an international inception cohort. Ann Rheum Dis 2013:72:1308-14.

13 Hochberg MC. Updating the American College of Rheumatology revised criteria for the classification of systemic lupus erythematosus. Arthritis Rheum 1997;40:1725

14 Alberti KG, Eckel RH, Grundy SM, et al. Harmonizing the metabolic syndrome: a joint interim statement of the International Diabetes Federation Task Force on Epidemiology and Prevention; National Heart, Lung, and Blood Institute; American Heart Association; World Heart Federation; International Atherosclerosis Society; and international association for the Study of Obesity. Circulation 2009;120:1640-5

15 Gladman DD, Ibanez D, Urowitz MB. Systemic lupus erythematosus disease activity index 2000. J Rheumatol 2002;29:288-91.
16 Gladman D, Ginzler E, Goldsmith C, et al. The development and initial validation of the Systemic Lupus International Collaborating Clinics/American College of Rheumatology damage index for systemic lupus erythematosus. Arthritis Rheum 1996:39:363-9.

17 Rojas RA, guilar-Salinas CA, Jimenez-Corona A, et al. Metabolic syndrome in Mexican adults: results from the National Health and Nutrition Survey 2006. Salud Publica Mex 2010;52(Suppl 1):S11-18.

18 Lorenzo C, Williams K, Hunt KJ, et al. The National Cholesterol Education Program —Adult Treatment Panel III, International Diabetes Federation, and World Health Organization definitions of the metabolic syndrome as predictors of incident cardiovascular disease and diabetes. Diabetes Care 2007:30:8-13.

19 Parker B, Ahmad Y, Shelmerdine J, et al. An analysis of the metabolic syndrome phenotype in systemic lupus erythematosus. Lupus 2011;20:1459-65.

20 Bellomio V, Spindler A, Lucero $E$, et al. Metabolic syndrome in Argentinean patients with systemic lupus erythematosus. Lupus 2009;18:1019-25.

21 Sabio JM, Zamora-Pasadas M, Jimenez-Jaimez J, et al. Metabolic syndrome in patients with systemic lupus erythematosus from Southern Spain. Lupus 2008; 17:849-59.

22 Negron AM, Molina MJ, Mayor AM, et al. Factors associated with metabolic syndrome in patients with systemic lupus erythematosus from Puerto Rico. Lupus 2008;17:348-54

23 Chung CP, Avalos I, Oeser A, et al. High prevalence of the metabolic syndrome in patients with systemic lupus erythematosus: association with disease characteristics and cardiovascular risk factors. Ann Rheum Dis 2007;66:208-14.

24 Telles R, Lanna C, Ferreira G, et al. Metabolic syndrome in patients with systemic lupus erythematosus: association with traditional risk factors for coronary heart disease and lupus characteristics. Lupus 2010;19:803-9.

25 Sabio JM, Vargas-Hitos J, Zamora-Pasadas M, et al. Metabolic syndrome is associated with increased arterial stiffness and biomarkers of subclinical atherosclerosis in patients with systemic lupus erythematosus. J Rheumatol 2009:36:2204-11

26 Ryan MJ, McLemore GR Jr, Hendrix ST. Insulin resistance and obesity in a mouse model of systemic lupus erythematosus. Hypertension 2006:48:988-93.

27 Condon MB, Ashby D, Pepper RJ, et al. Prospective observational single-centre cohort study to evaluate the effectiveness of treating lupus nephritis with rituximab and mycophenolate mofetil but no oral steroids. Ann Rheum Dis 2013;72:1280-6.

28 Wallace DJ, Metzger AL, Stecher VJ, et al. Cholesterol-lowering effect of hydroxychloroquine in patients with rheumatic disease: reversal of deleterious effects of steroids on lipids. Am J Med 1990;89:322-6.

29 Penn SK, Kao AH, Schott LL, et al. Hydroxychloroquine and glycemia in women with rheumatoid arthritis and systemic lupus erythematosus. J Rheumatol 2010;37:1136-42. 